\title{
Prostatectomia Transvesical X Prostatectomia a Millin: Revisão de literatura
}

\author{
Transvesical prostatectomy X Millin prostatectomy: Literature review
}

Tadeu José Fontenele Leite Campos"

\author{
Hospital Geral de Fortaleza, Fortaleza, Ceará, Brasil *Autor para correspondência. E-mail: tadeucampos7@gmail.com
}

\begin{abstract}
Resumo: Introdução: A hiperplasia prostática benigna (HPB) é uma das doenças que mais comumente afeta o homem adulto, gerando importante prejuízo na qualidade de vida destes. Atualmente, existem diversos tratamentos para a HPB, incluindo os medicamentos, endoscópicos e minimamente invasivos. Porém a cirurgia aberta, na forma da prostatectomia, ainda possuem seu espaço no tratamento de pacientes selecionados, principalmente nos serviços públicos de saúde. Revisão: Objetivou comparar, com base na literatura, as cirurgias de prostatectomia transvesical e prostatectomia a Millin para o tratamento da HPB, no que diz respeito a vantagens/desvantagens, indicações e complicações pós-operatórias. Discussão: Com base na literatura, não se pode afirmar a superioridade de uma das técnicas. Elas apresentam suas vantagens e desvantagens, sendo a escolha entre elas, principalmente, uma decisão do cirurgião com base em sua experiência. Considerações finais: Em países subdesenvolvidos, como no Brasil, a prostatectomia aberta ainda tem importante papel no tratamento de pacientes com próstatas volumosas. Permanece importante o estudo, discussão, aprimoramento e disseminação da técnica de prostatectomia aberta aos residentes de urologia em formação.
\end{abstract}

Palavras-chave: Complicações peroperatórias, Hiperplasia prostática, Prostatectomia retropúbica, Prostatectomia suprapúbica.

\begin{abstract}
Introduction: The benign prostatic hyperplasia (BPH) is one of the most commonly diseases that affects adult men, causing significant damage to their quality of life. Currently, there are several treatments for BPH, including medications, endoscopic and minimally invasive. However, open surgery, in the form of prostatectomy, still has its space in the treatment of selected patients, mainly in public health services. Review: The objective was to compare, based on the literature, the transvesical prostatectomy and Millin prostatectomy surgeries for the treatment of $\mathrm{BPH}$, with regard to advantages / disadvantages, indications and postoperative complications. Discussion: Based on the literature, we cannot affirm the superiority of one of the techniques. They have their advantages and disadvantages, and the choice among them is mainly a decision of the surgeon based on his experience. Final considerations: In underdeveloped countries, such as Brazil, open prostatectomy still plays an important role in the treatment of patients with large prostates. The study, discussion, improvement and dissemination of the open prostatectomy technique to urology residents in training remains important.
\end{abstract}

Keywords: Peroperative complications, Prostatic hyperplasia, Retropubic prostatectomy, Suprapubic prostatectomy.

\section{Introdução}

A hiperplasia prostática benigna (HPB) é considerado a neoplasia benigna mais comum do homem, sendo uma condição inerente ao processo de envelhecimento. Bastante comum a partir da sexta década de vida, acarreta impacto negativo relevante na qualidade de vida do homem idoso (Nardozza Júnior et al., 2010).

Atualmente, dispomos de diversos métodos para o tratamento da HPB, agrupados, basicamente em quatro categorias: observação, terapia medicamentosa, terapias minimamente invasivas e o tratamento cirúrgico aberto. Para escolha do método mais adequado, deve ser feita uma avaliação individualizada de cada paciente, considerando seus sintomas, comorbidades e experiência do cirurgião (Nardozza Júnior et al., 2010; Roehrborn, 2002).

Com relação ao tratamento cirúrgico, a escolha do acesso por via aberta ou endoscópica vai depender do tamanho da próstata, disponibilidade de materiais e comorbidades do paciente (Paranhos et al., 2011)

A cirurgia aberta pode, basicamente, ser realizada por duas técnicas: suprapúbica transvesical ou retropúbica a Millin. Esse procedimento é reservado para próstatas de dimensões maiores e é considerada a 
técnica com melhores resultados a longo prazo, com taxa de reintervenção de $2 \%$. Como desvantagem, temos um maior índice de complicação e maior tempo de hospitalização (Reich et al., 2006; Nardozza Júnior et al., 2010).

Apesar dos avanços recentes e desenvolvimento de novas técnicas cirúrgicas para tratamento de próstatas volumosas, como a enucleação endoscópica com laser, a prostatectomia simples aberta continua tendo importante papel para tratamento dos pacientes do serviço público de saúde que não possuem acesso a essas novas tecnologias.

Dessa forma, realizamos uma revisão de literatura comparativa entre as duas técnicas mais utilizadas de prostatectomia simples aberta, trasnvesical e retropubica, com intuito de avaliar se alguma apresenta melhor perfil de segurança e morbidade.

\section{Revisão}

O referido estudo foi desenvolvido com base nas evidências científicas da literatura no que diz respeito a prostatectomia aberta para tratamento da HPB. O levantamento bibliográfico foi realizado nas bases de dados MEDLINE-Indez Medicus (Medical Literature Analysisn and Retrieval System Online), LILACS - BIREME (Bases de dados da literatura Latino Americana, em Ciências de Saúde) e SciELO (Scidentific Eletrônica Library Online), além de livros e revistas de Urologia.

Foi realizado levantamento das produções cientificas a respeito da HPB e seus tratamentos, com ênfase na cirurgia aberta. Os critérios de inclusão das fontes bibliográficas foram trabalhos publicados no período de 1991 a 2016, que estivessem disponíveis em texto completo, em português ou inglês.

$\mathrm{O}$ aumento do volume prostático e a atividade alfa-adrenérgica aumentada no estroma prostático observados no paciente com HPB, pode determinar obstrução infravesical e, consequentemente, mas não sempre, sintomas do trato urinário inferior (STUIs). Tais sintomas impactam significativamente na vida do homem idoso (Rhoden, 2009; Danilovic \& Claro, 2015).

Estima-se uma prevalência de HPB histológica de 10\%, 50\% e 90\%, respectivamente, aos 25,60 e 80 anos. A HPB clínica, porém, é menos frequente, apesar de ainda ser bastante prevalente. $60 \%$ dos pacientes na sétima década de vida apresentam algum sintoma decorrente da HPB. Aos 55 anos, aproximadamente 25\% dos homens relatam STUIs (Nardozza Júnior et al., 2010; Arrighi et al., 1991).

Além dos clássicos e inespecíficos sintomas da HPB, o paciente pode também apresentar complicações dessa patologia, que, para muitos autores são indicação formal de tratamento cirúrgico. Entre essas, podemos citar a hematúria macroscópica, a insuficiência renal pós- renal, infecções de repetição, litíase vesical e retenção urinária (Srougi, 1995).

O objetivo do tratamento da HPB consiste basicamente em aliviar sintomas do paciente, melhorando sua qualidade de vida, e prevenir complicações (Nardozza Júnior et al., 2010).

Atualmente, existem diversas modalidades de tratamento para o paciente com HPB, desde o simples acompanhamento, tratamento medicamentoso, procedimentos minimamente invasivos e cirurgia aberta. A escolha depende da experiência do cirurgião, sintomas do paciente, comorbidades, tamanho da próstata e disponibilidade do serviço. Focaremos no tratamento cirúrgico, mais especificamente, na prostatectómica aberta.

Dentro dessa modalidade de tratamento, podemos incluir a incisão transuretral da próstata, a ressecção transuretral da próstata (RTUp), a enucleação endoscópica com laser e a prostatectomia laparoscópica, robótica e aberta (Srougi et al., 2008; Lepor et al., 1996; Emberton et al., 2008).

A prostatectomia aberta foi o primeiro procedimento cirúrgico desenvolvido para o tratamento do HPB e, assim, persistiu por vários anos. Sem dúvida, esse procedimento perdeu bastante espaço com o advento das técnicas endoscópicas que hoje dominam a terapêutica da HPB. Porém permanece como procedimento importante na urologia para o tratamento de adenomas mais volumosos (Dall'Oglio et al., 2006).

As indicações absolutas de tratamento cirúrgico da HPB são basicamente as complicações decorrentes da patologia. Classicamente, a escolha se tal tratamento cirúrgico vai ser endoscópico ou aberto depende, basicamente, da disponibilidade do serviço, da experiência do cirurgião e do tamanho da próstata.

A cirurgia aberta pode ser realizada, basicamente, por duas técnicas: a prostatectomia suprapúbica transvesical (PTV) de Fuller - Freyer ou a retropúbica a Millin modificada por Srougi. A escolha entre as técnicas depende da preferência do cirurgião e de patologias vesicais associadas (Gratzke et al., 2007).

Ainda bastante utilizada nos dias atuais, a PTV tem sua indicação principalmente nas próstatas volumosas com patologia vesical, tais como cálculos maiores que $2 \mathrm{~cm}$ e divertículos.

Descrita em 1947, por Millin e modificada em 2003 por Srougi, a prostatectomia retropúbica foi desenvolvida com o intuito principal de diminuir as complicações hemorrágicas observadas na PTV, além de ser realizada sem a abertura da bexiga, o que reduz a incidência de fístulas (Srougi et al., 2003). 
Quando há concomitância entre HPB e patologias vesicais, tais como cálculos maiores que $2 \mathrm{~cm}$ e divertículos, tem - se preferência pelo acesso transvesical. Nos casos de adenoma com logo mediano volumoso, diversos cirurgiões preferem a PTV devido a visualização direta dos meatos ureterais, evitando lesões que podem ocorre no acesso retro púbico (Luttwak et al., 1997).

Como desvantagens, temos a não visualização, durante a PTV, do ápice prostático e de toda a loja prostática durante a hemostasia. Temos ainda maior número de complicações hemorrágicas no perioperatório com necessidade de transfusão. Por fim, a abertura da bexiga como tempo do procedimento cirúrgico, coloca o paciente sobre maior risco de desenvolver fistula urinaria no pós-operatório (Paranhos et al., 2011).

Mais recente, a técnica proposta por Millin ganhou bastante espaço no tratamento da HPB. Tem vantagem de permitir a visualização até do ápice prostático e de toda a loja prostática para revisão de hemostasia após a enucleação do adenoma.

Essa técnica foi desenvolvida com intuito de diminuir as complicações hemorrágicas da PTV, por permitir maior controle do sangramento por ligaduras vasculares antes da enucleação e por permitir revisão de hemostasia da loja prostática após a enucleação. Por não abrir a bexiga, tal técnica também tem menor índice de fistula urinária (Danilovic \& Claro, 2015).

A PTV e a prostatectomia a Millin apresentam perfis e índices diferentes de complicações pós-operatórias. Nesse trabalho vamos nos focar nas complicações urológicas decorrentes das duas técnicas cirúrgicas, porém devemos lembrar que esses pacientes apresentam frequentemente complicações não urológicas, tais como trombose venosa profunda, infarto agudo do miocárdio, entre outras (Stutzman, 2007).

As complicações hemorrágicas sempre foram as mais preocupantes em pacientes submetidos a PTV, a despeito do avanço da técnica e de sua hemostasia ao longo dos anos. Temos, recentemente, uma incidência de sangramento importante de 11,6\%, com necessidade de transfusão em 7,5 \% dos pacientes, determinando uma taxa de necessidade de revisão cirúrgica de 3,7\% (Gratzke et al., 2007).

No estudo de Elshal et al. (2013) foi observado uma alteração significativa no nível médio de hemoglobina no sangue, hematócrito e nível médio de creatinina sérica entre o pré-operatório (13,7g, 40,9\% e 1,1 mg / dL, respectivamente) e imediatamente após a PTV $(10.9 \mathrm{~g}, 33.1 \%$ e $1.55 \mathrm{mg} / \mathrm{dL})$.

Carneiro et al. (2016), em um estudo com 65 pacientes submetidos a prostatectomia evidenciou uma estimativa peri-operatória de perda de sangue na PTV de $927 \mathrm{ml}$ e uma média de variação de Hb após 48 horas 13,78 a 10,49mg / dL. A transfusão de sangue durante a cirurgia foi necessária para três pacientes tratados por PTV.

Outro estudo, em mais de 60 pacientes, demonstrou um sangamento transoperatório de aproximadamente $640 \mathrm{ml}$, variando de 200 a $1500 \mathrm{ml}$, nos pacientes submetidos a PTV (Dall’Oglio et al., 2006).

O extravasamento de urina, com possivel formação de fístula urinária, pode ser motivo de preocupação no pós-opertarorio imediato, através da rafia da bexiga urinária ou pericistostomia. Porém, tal condição resolvese espontaneamente na grande maioria dos casos apenas com caterismo vesical mais prolongado (Paranhos et al., 2011).

Elshal et al. (2013) em seu estudo retrospectivo identificou uma taxa de extravsamaneto de urina / fistula urinária de aproximadamente $4,9 \%$ nos pacientes submetidos a PTV, sendo todos conduzidos com drenagem prolongada da bexiga.

A incontinência urinária por insuficiência esfincteriana associada a lesão iatrogênica do esfincter externo uretral é uma condição rara, ocorrendo em casos de avulsão de grandes adenomas. Sua incidência geral na literatura varia de $0,5 \%$ a $8 \%$ (Roehrborn, 2010).

No estudo de Carneiro et al. (2016), de 32 pacientes submetdos a PTV, nenhum desenvolveu quadro de incontinência urinária como complicação tardia.

Outro grupo de complicações pós-operatórias da prostatectomia suprapúbica, são as infecciosas, incluindo a infecção de ferida operatória e a infecção do trato urinário (ITU). Ambas podem ser evitadas com a combinação de técnica adequada e uso de antibioticoprofilaxia. Nesse sentido, todos os pacientes que estão em pré-operatório de PTV, deve colher ainda no ambulatório uma urocultura e tratar a infecção urinária se existente. Em média essas complicações ocorrem em 3\% desses paciente (Stutzman, 2007).

Carneiro et al (2016) observou, em seu estudo, dos 32 pacientes submetidos a PTV, a ocorrência de ITU em 4 e nenhum caso de infecção de ferida operatória.

Em um estudo observacional com 202 pacientes submetidos a cirurgia de prostatectomia transvesical, evidenciou-se uma taxa de infecção de sítio cirurgico de 3,94\% e uma taxa de ITU de 5,44\% (Inzunza, 2014).

A estenose de uretra e a esclerose do colo vesical são raras na PTV, ocorrendo mais comumente nas técnicas de ressecção endoscópica. Tais complicalções apresentam uma taxa média de ocorrência na literatura de 3 a 6\% (Louis et al., 2006). 
Dos 32 pacientes subemtidos a PTV do estudo de Carneiro et al (2016), 2 apresentaram estenose de uretra e 1 esclerose do colo vesical como complicação tardia. No estudo de Inzunza (2014), a taxa de estenose de uretra foi de $1,48 \%$.

A prostatectomia a Millin foi desenvolvida com intuito de diminuir as complicações da PTV, principalmente no que diz respeito ao sangramento perioperatório e necessidade de transfusão. Essa técnica apresenta complicações semelhantes as descritas acima com relação a PTV, porém com taxas diferentes (Louis et al., 2006).

Em um estudo prospectivo de mais de 60 pacientes, comparando as técnicas de PTV e prostactectomia a Millin, foi observado uma diferença significativa do sangramento transoperatório, sendo de $640 \mathrm{ml}$ (200 a $1500 \mathrm{ml}$ ) e 362 $\mathrm{ml}(50$ a $700 \mathrm{ml})$, respectivamente. A queda dos níveis de hemoglobina no pós-operatório imediato foi significativamente maior nos pacientes submetidos a PTV (Dall'Oglio et al., 2006).

Alguns estudos, no entanto, não demonstram essa superioridade da cirurgia a Millin no que diz respeito as complicações hemorrágicas. Carneiro et al. (2016) observou um sangramento semelhante entre as duas técnicas, porém com necessidade de transfusão maior na PTV.

Ainda no estudo de Carneiro et al. (2016), para próstatas com mais $100 \mathrm{~g}$, o sangramento da cirurgia a Millin foi significativamente maior do que o da PTV, sendo $1313 \mathrm{ml}$ e $863 \mathrm{ml}$, respectivamente. O autor sugere como causa para essa diferença, a dificuldade de controle vascular dos pedículos laterais em grandes prostátas.

Com relação as complicações infecciosas da prostatectomia a Millin, temos um estudo realizado na Bavaria, com 902 pacientes, onde ocorre infecção urinária em 5,1\% (Reich et al., 2008).

No estudo de Taha et al. (2011), realizado com 96 pacientes subemtidos a prostatectomia retropúbica, identificou-se infecção de ferida operatória em 6,3\%. Nesse mesmo estudo, a taxa de fistula urinária foi de $4,2 \%$ e a de estenose de uretra foi de $5,2 \%$

\section{Discussão}

Apesar do avanço das técnicas endoscópicas e minimamente invasivas no tratamento da HPB, uma parcela significativa de pacientes ainda é submetida ao tratamento através da prostatectomia aberta, principalmente, no sistema público de saúde.

Millin desenvolveu a técnica retropúbica com o objetivo de minimizar as complicações observadas na técnica transvesical. Com base nos estudos presentes na literatura, podemos observar que as complicações das duas técnicas são bastante semelhantes, incluindo sangramento perioperatório, complicações infecciosas, fístulas, incontinência urinária, estenose de uretra, entre outras.

A diferença entre as duas técnicas está na taxa das complicações em cada uma. Podemos observar que, na maioria dos estudos, as complicações hemorrágicas são maiores na PTV. Porém, alguns estudos demonstram taxas de sangramento semelhante e outro mostra sangramento maior na cirurgia a Millin para próstatas com mais de 100g.

Por fim, fica claro que não podemos definir uma técnica como superior. As duas ainda são bastante usadas, cada uma com suas vantagens e desvantagens. Sendo escolhidas muito a depender da experiência do cirurgião que, sem dúvida interfere de forma significativa nas taxas de complicações, o que deve justificar as discrepâncias de alguns estudos da literatura.

\section{Considerações finais}

Em países subdesenvolvidos, como no Brasil, a prostatectomia aberta ainda tem importante papel no tratamento de pacientes com próstatas volumosas. O acesso destes pacientes a novas tecnologias minimamente invasivas ainda parece estar longe. Dessa forma, permanece importante o estudo, discussão, aprimoramento e disseminação da técnica de prostatectomia aberta aos residentes de urologia em formação.

\section{Referências}

Elshal, A. M., El-Nahas, A. R., Barakat, T. S., Elsaadany, M. M., \& El-Hefnawy, A. S. 2013. Transvesical open prostatectomy for benign prostatic hyperplasia in the era of minimally invasive surgery: perioperative outcomes of a contemporary series. Arab Journal of Urology, 11(4), 362-368.

Arrighi, H. M., Metter, E. J., Guess, H. A., \& Fozzard, J. L. 1991. Natural history of benign prostatichyperplasia and risk of prostatectomy: the Baltimore longitudinal study of aging. Urology, 38(1), 4-8. 
Carneiro, A., Sakuramoto, P., Wroclawski, M. L., Forseto, P. H., Julio, A. D., Bautzer, C. R. D., ... \& Pompeo, A. C. L. 2016. Open suprapubic versus retropubic prostatectomy in the treatment of benign prostatic hyperplasia during resident's learning curve: a randomized controlled trial. International Brazilian Journal of Urology, 42(2), 284-292.

Dall'Oglio, M. F., Srougi, M., Antunes, A. A., Crippa, A., \& Cury, J. 2006. An improved technique for controlling bleeding during simple retropubic prostatectomy: a randomized controlled study. BJU International, 98(2), 384-387.

Danilovic, A., \& Claro, J. F. A. 2015. Excelência e alta complexidade em Urologia. São Paulo, SP: AstraZeneca.

Emberton, M., Cornel, E. B., Bassi, P. F., Fourcade, R. O., Gomez, J. M. F., \& Castro, R. 2008. Benign prostatic hyperplasia as a progressive disease: a guide to the risk factors and options for medical management. International Journal of Clinical Practice, 62(7), 1076-1086.

Rhoden, E. L. 2009. Urologia no consultório. ArtMed. Porto Alegre.

Gratzke, C., Schlenker, B., Seitz, M., Karl, A., Hermanek, P., Lack, N., ... \& Reich, O. 2007. Complications and early postoperative outcome after open prostatectomy in patients with benign prostatic enlargement: results of a prospective multicenter study. The Journal of Urology, 177(4), 1419-1422.

Inzunza, N., \& Antonio, J. 2014. Manejo quirúrgico de la hiperplasia prostática benigna y evaluación de eventos adversos según Clavien. Experiencia en Hospital de Villarrica. Revista Chilena de Urologia, 79(4) $34-40$.

Lepor, H., Williford, W. O., Barry, M. J., Brawer, M. K., Dixon, C. M., Gormley, G., ... \& Padley, R. J. 1996. The efficacy of terazosin, finasteride, or both in benign prostatic hyperplasia. New England Journal of Medicine, 335(8), 533-540.

Louis, R. K., Alan, J. W., \& Andrew, C.N. 2006. Campbell-Walsh Urology. Philadelphia, US: Elsevier.

Luttwak, Z., Lask, D., Abarbanel, J., Manes, A., Paz, A., \& Mukamel, E. 1997. Transvesical prostatectomy in elderly patients. The Journal of Urology, 157(6), 2210-2211.

Nardozza Júnior, A., Zerati Filho, M., \& Reis, R. B. 2010. Urologia Fundamental. Sociedade Brasileira de Urologia. São Paulo.

Paranhos, M., Antunes, A. A., \& Dall’oglio, M. F. 2011. Cirurgia Próstatica: Técnicas e Truques. São Paulo, SP: DOC.

Reich, O., Gratzke, C., Bachmann, A., Seitz, M., Schlenker, B., Hermanek, P., ... \& Urology Section of the Bavarian Working Group for Quality Assurance. 2008. Morbidity, mortality and early outcome of transurethral resection of the prostate: a prospective multicenter evaluation of 10,654 patients. The Journal of Urology, 180(1), 246-249.

Roehrborn, C. G. 2002. Reducing the risk of benign prostatic hyperplasia progression. Reviews in Urology, 4(Suppl 5), S29-S38.

Srougi, M., Dall'oglio, M. F., Bomfim, A. D. C., Andreoni, C., Cury, J., \& Ortiz, V. 2003. An improved technique for controlling bleeding during simple retropubic prostatectomy. BJU International, 92, 813-7.

Srougi, M., Ribeiro, L. A., Piovesan, A. C., Colombo, J. R., \& Nesrallah, A. 2008. Doenças da próstata. Revista de Medicina, 87(3):166-77.

Srougi, M. 1995. Hiperplasia prostática. Rio de Janeiro, RJ: Record.

Stutzman, R. E. 2007. Section IV, Prostate: Open prostatectomy. In S. D. Graham \& T. E. Keane. Glenn's Urologic Surgery (7th ed.). Philadelphia, US: Lippincott Williams \& Wilkins.

Reich, O., Gratzke, C., \& Stief, C. G. 2006. Techniques and long-term results of surgical procedures for BPH. European urology, 49(6), 970-978.

\section{Minicurrículo}

Tadeu José Fontenele Leite Campos. Médico formado pela Universidade Federal de Campina Grande, cirurgião pelo Hospital Geral Waldemar de Alcântara e urologista pelo Hospital Geral de Fortaleza. Vinculado ao corpo clínico de urologistas do Hospital Geral de Fortaleza. 
Como citar: Campos, T.J.F.L. 2021. Prostatectomia Transvesical X Prostatectomia a Millin: Revisão de literatura. Pubsaúde, 5, a113. DOI: https://dx.doi.org/10.31533/pubsaude5.a113

Recebido: 9 dez. 2020.

Revisado e aceito: 5 jan. 2021.

Conflito de interesse: os autores declaram, em relação aos produtos e companhias descritos nesse artigo, não ter interesses associativos, comerciais, de propriedade ou financeiros que representem conflito de interesse.

Licenciamento: Este artigo é publicado na modalidade Acesso Aberto sob a licença Creative Commons Atribuição 4.0 (CC-BY 4.0). 